\title{
La maquette, un outil au service d'une éducation aux risques
}

Scale models, a tool in teaching risk management

La maqueta una herramienta para la educación sobre los riesgos

David Bédouret, Raphaël Chalmeau, Christine Vergnolle-Mainar, Marie Pierre Julien, Jean-Yves Léna et Anne Calvet

\section{(2) OpenEdition}

\section{Journals}

Édition électronique

URL : http://journals.openedition.org/mappemonde/4572

DOI : $10.4000 /$ mappemonde.4572

ISSN : 1769-7298

Éditeur

UMR ESPACE

Référence électronique

David Bédouret, Raphaël Chalmeau, Christine Vergnolle-Mainar, Marie Pierre Julien, Jean-Yves Léna et Anne Calvet, "La maquette, un outil au service d'une éducation aux risques », Mappemonde [En ligne], 129 | 2020, mis en ligne le 10 novembre 2020, consulté le 26 novembre 2020. URL : http:// journals.openedition.org/mappemonde/4572 ; DOI : https://doi.org/10.4000/mappemonde.4572

Ce document a été généré automatiquement le 26 novembre 2020. 


\title{
La maquette, un outil au service d'une éducation aux risques
}

\author{
Scale models, a tool in teaching risk management \\ La maqueta una herramienta para la educación sobre los riesgos
}

David Bédouret, Raphaël Chalmeau, Christine Vergnolle-Mainar, Marie

Pierre Julien, Jean-Yves Léna et Anne Calvet

\section{Introduction}

1 Les maquettes d'un lieu sont des outils de représentation du réel utilisés de longue date. Elles donnent à voir les reliefs et les formes d'occupation de l'espace, à une échelle permettant à l'observateur d'en avoir une vision globale, surplombante et en adoptant différents angles d'observation. Les plans-reliefs en vogue dès l'époque moderne dans une perspective militaire en sont une des traductions. Mais depuis, bien d'autres usages se sont développés, notamment comme moyen de communication pour des projets d'aménagement, mais aussi en tant qu'outil pédagogique dans l'enseignement de la géographie et des sciences de la Terre. Ainsi, Lucien Lefèvre (1947) a été pionnier dans la réalisation par des élèves de sixième d'un plan-relief du milieu local afin de synthétiser les observations faites lors d'enquêtes sur le terrain.

$2 \mathrm{Au}$ tournant des $\mathrm{XX}^{\mathrm{e}}$ et $\mathrm{XXI}^{\mathrm{e}}$ siècles, des travaux en didactique de la géographie ont également mis en évidence l'intérêt des maquettes, notamment pour travailler l'acquisition de repères spatiaux avec de jeunes enfants (Considère et Griselin, 1997 ; Leroyer, 2005; Godin, 2008). Elles sont en effet souvent mobilisées comme étape intermédiaire dans le passage du terrain au plan/carte et donc plus largement dans le basculement d'une vision oblique en 3D à une vision zénithale en 2D. Leur construction facilite en effet le passage de l'espace vécu à l'espace conçu (Godin, 2008), notamment en permettant la remobilisation d'un réel préalablement travaillé. Les maquettes apparaissent alors comme un levier pour passer de l'espace vécu à l'espace perçu (Leroyer, 2005). Mais au-delà de ces premiers apprentissages, elles permettent aussi de travailler sur les proportions et donc sur l'échelle ainsi que sur une symbolisation 
préfigurant une légende (Considère et Griselin, 1997). En sciences, à l'école primaire, des maquettes sont aussi utilisées en tant qu'un des outils de modélisation du réel afin de l'expliquer, en l'occurrence un modèle physique (Walliser, 1977) reposant sur une forte analogie avec le réel (Merle, 2000).

Des travaux récents portant sur l'usage de maquettes dans le cadre d'ateliers-débats à visée prospective avec des habitants (Buyck et al., 2016 ; Romero et al., 2017) peuvent être des points d'appui pour diversifier l'usage scolaire des maquettes. En effet, ces travaux mettent l'accent sur le potentiel de dialogue dont les maquettes sont porteuses. Elles sont un médium, un support concret de discussions qui permet de confronter les regards, de faire émerger la complexité et ainsi de faire évoluer les représentations initiales. Mais Buyck et al. (2016) soulignent aussi l'intérêt d'associer les habitants à la réalisation de maquettes : elles sont alors à la fois "un espace déjà là et en devenir " permettant de poser des questions et surtout de prendre des décisions.

Ces travaux montrent l'intérêt des maquettes tant pour approcher la complexité d'un territoire que pour en discuter le devenir. Dans le cadre d'une éducation aux risques, nous avons mobilisé la maquette comme outil heuristique favorisant une réflexion spatiale et citoyenne et nous en présentons les usages faits par les élèves. Après avoir replacé le cadre théorique, la méthodologie et le protocole, nous énoncerons les résultats que nous discuterons.

\section{Cadre théorique : une modélisation pour une éducation aux risques}

5 Le champ de l'éducation aux risques est en émergence, notamment quand il est considéré sous l'angle de la prévention et de la réduction de la vulnérabilité plutôt que sous celui de l'acquisition de réactions appropriées en cas de catastrophe. Ce type d'éducation va au-delà de l'enseignement des risques, car elle ne s'arrête pas à la description et à l'explication des mécanismes des aléas et de leurs conséquences sur les sociétés. Elle est un projet sociétal cherchant à augmenter notre résilience et dépasse alors le cadre scolaire. Ainsi, cette éducation aux risques peut trouver des appuis dans les démarches d'ancrage territorial et nécessite de développer des démarches éducatives impliquant fortement les enfants et allant au-delà des propos médiatiques inscrits dans le sensationnel (Antoine, 2010, p. 204). Ceux-ci sont souvent relayés dans les manuels scolaires (Frézal-Leininger, 2014) et participent de la construction d'une appréhension déterritorialisée des risques.

\section{Appréhender son territoire pour une éducation aux risques}

6 L'éducation, entendue dans sa définition la plus large, apparait comme un rempart face à des évènements catastrophiques naturels (Muttarak et Lutz, 2014) et représente «le facteur social et économique le plus important de réduction de la vulnérabilité aux catastrophes naturelles » (Striessnig et al., 2013, p. 6).

Une éducation aux risques doit commencer par la perception, la conscience du (des) risque(s), rendues possibles par le vécu et/ou par la culture du groupe dans lequel l'élève vit. Ce dernier les acquiert par la connaissance du territoire où il vit et sa propre expérience des catastrophes locales (Tanner, 2010). Il s'avère ainsi essentiel de 
contextualiser l'enseignement des thématiques sur les risques en prenant en compte des exemples locaux à proximité des élèves. Une éducation aux risques s'intègre donc dans une éducation plus large "au territoire». Ce dernier apparait alors comme un levier pour la construction d'une implication citoyenne, car porteur de valeurs et «facteur d'ancrage social» (Blanc-Maximin et Floro, 2017 ; Barthes et al., 2019). Pour travailler le lien entre l'élève et son territoire, l'approche développée doit intégrer les caractéristiques locales (espaces et acteurs locaux) et doit forcément passer par une éducation dans le territoire (Dussaux, 2017). Dans cette perspective, la finalité est d'aider l'élève à devenir un véritable acteur local : un acteur capable d'identifier et de comprendre les enjeux relatifs à son lieu de vie, de se situer dans les débats sociaux à leur sujet et, s'il le souhaite, d'agir (Bédouret et al., 2018).

8 L'éducation aux risques peut générer des effets positifs sur le comportement même de l'élève, mais également sur les personnes de son entourage direct voire de la communauté, via un transfert de savoirs (Clerveaux et Spence, 2009; Hoffmann et Muttarak, 2017; Lutz et al., 2014). Cependant, il est important que ce transfert puisse également se faire depuis les acteurs locaux jusqu'aux élèves. L'implication d'acteurs de la communauté locale dans des programmes scolaires permet ainsi une perception commune du risque, comme c'est le cas pour « Memo'Risks $»^{1}$. Ce programme a été mis en place dans le bassin versant de la Loire et réunit les autorités locales et les écoles afin d'étudier les situations locales de risque de catastrophe. En effet, une telle réflexion collective et locale sur un même territoire, accompagnée si possible d'actions, s'avère indispensable à la construction d'une «culture du risque » commune, qui doit permettre de réduire la vulnérabilité des sociétés (Blesius, 2013).

\section{La maquette, une entrée dans le territoire permettant d'impliquer l'enfant}

9 En sciences de la vie et de la Terre comme en géographie, la maquette est une forme de modèle au sens d'une « représentation formalisée et épurée du réel ou d'un système de relations » (Brunet et al., 1993, p. 334). Dans l'usage scolaire, maquette et modèle se fondent souvent en un même outil heuristique permettant aux élèves de (se) représenter un phénomène inaccessible directement, pour le comprendre. Mais l'un et l'autre reposent sur des épistémologies différentes liées au contexte disciplinaire de leur usage : le modèle est privilégié en SVT et la maquette en géographie. Leur usage croisé a été cependant plus développé dans l'enseignement des sciences qu'en géographie (Ferras, 1993). En sciences, il a débouché sur des avancées théoriques concernant les modèles qui peuvent être des points d'appui pour renouveler l'usage de la maquette dans le cadre d'une réflexion territoriale menée en classe.

«Les modèles doivent être présentés comme des outils approximatifs et non comme des réalités intangibles» (Giordan et De Vecchi, 1987, p. 203). Ainsi, en didactique des sciences, un des enjeux de l'usage de maquette (ou de modèles dans un sens plus large) consiste à « mettre en évidence le fait que les modèles ne correspondent pas à la réalité ${ }^{2}$, mais sont les instruments d'explication et de prévision produits en vue de mieux comprendre les phénomènes ou les problèmes rencontrés " (Giordan et De Vecchi, 1987, p. 203). Cet enjeu implique une nécessité pédagogique dans l'enseignement des sciences: il faut que les élèves puissent confronter le modèle au réel et le réel au 
modèle. Cet aller-retour permet d'éprouver les limites du modèle et d'en construire le domaine de validité.

11 Pour définir un modèle, on peut s'appuyer sur la définition déjà ancienne proposée par Birou (1966): un modèle est un "système physique, mathématique ou logique représentant les structures essentielles d'une réalité et capable à son niveau d'en expliquer ou d'en reproduire dynamiquement le fonctionnement». En didactique des sciences, Host (1989, p. 204) définit le modèle comme « toute représentation matérielle, iconique ou symbolique faite en vue d'une explication, c'est-à-dire, qui reproduit certains aspects de l'objet étudié pour comprendre son fonctionnement et déduire des propriétés nouvelles ». Les visées de l'utilisation de modèles sont diverses comme le souligne Drouin (1988) : comprendre, expliquer, prévoir, calculer, manipuler, formuler des analogies, communiquer, rendre pensable ce qui est difficile à cerner, voici donc les fonctions qui se dégagent des divers modèles que l'on peut rencontrer. On retrouve à l'école primaire deux visées principales de l'utilisation ou de la construction de modèles, certains modèles semblent avoir pour fonction principale d'expliquer, d'autres plutôt de prévoir, c'est-à-dire d'anticiper par la pensée le déroulement d'un phénomène, à travers la théorie interprétative que constitue le modèle (Drouin, 1988).

En sciences, un modèle peut être représenté par un objet matériel comme une maquette, un schéma simplificateur sous forme d'image réaliste, une analogie avec ou sans figuration (Bodur et Guichard, 2006). Pierrard (1988), dans une activité de modélisation en astronomie avec des élèves de $\mathrm{CM}$, indique que trois caractéristiques des modèles en sciences apparaissent dans l'activité de classe : ce sont des substituts du réel; il y a analogie (réversible) entre le modèle et l'espace matériel; différents modèles peuvent expliquer un même phénomène. Dans ce contexte, la maquette est considérée comme un "modèle physique ", au sens de Walliser (1977), puisqu'il s'agit d'une maquette utilisant des objets concrets homothétiques des objets réels (Merle, 2000). L'auteure indique également que si les modèles utilisés par les élèves sont des maquettes, ces modèles sont donc caractérisés par leur aspect figuratif, leur fonction essentielle étant une fonction de représentation (Martinand, 1992). Cette représentation repose sur des choix où seuls les aspects les plus pertinents de la réalité sont pris en compte, ils sont alors symbolisés et réassociés les uns aux autres dans l'élaboration du modèle. Ce processus cognitif est une véritable reconstruction.

Dans ce contexte, la dimension figurative de la maquette est retenue en tant qu'élément de compréhension et de reconstruction du réel, mais aussi en tant qu'objet manipulable permettant de s'affranchir du réel actuel et de penser son devenir.

\section{Méthodologie et protocole : une ingénierie pour une éducation aux risques}

Les élèves du cycle 2 et du cycle 3 de l'école publique d'Esquièze-Sère, localisée dans les Hautes-Pyrénées constituent les sujets de cette étude. Ils sont organisés en deux classes, une par cycle, avec dix élèves de 6 à 8 ans pour le cycle 2 et treize élèves de 8 à 10 ans pour le cycle 3 . Une séquence pédagogique portant sur l'éducation aux risques, co-construite avec les enseignants de ces deux classes ${ }^{3}$, fournit le contexte de cette étude. Le travail spécifique sur la réalisation de la maquette est conduit par cinq binômes de cycle 2 et six groupes de deux à trois élèves de cycle 3 . 

la trame générale du déroulement de la séquence est présentée ci-dessous en vue d'indiquer la nature et le contexte des recueils des données présentées et analysées dans cet article. risques dans la mesure où plusieurs aléas sont présents : notamment les crues, les avalanches, les glissements de terrain/chutes de blocs, les incendies et les séismes.

\section{Déroulement du projet pédagogique}

\section{Une ingénierie éducative organisée autour de trois temporalités}

17 La séquence pédagogique vise une découverte et une prise de conscience progressive des aléas et des risques du territoire dans lequel vivent les élèves. Une focale a été faite sur les inondations. Cette séquence s'articule autour de trois moments (présent, passé, futurs), prenant appui sur la démarche formalisée par notre équipe pour aborder avec des enfants les enjeux du futur de leur territoire (Vergnolle Mainar et al., 2016).

À propos du présent, les élèves confrontent leurs propres représentations (construction de nuages de mots et questionnaires) à la réalité du terrain (observation de la vallée du Gave, rencontre avec un chercheur du laboratoire GEODE au bord du Gave dans une zone aménagée après la crue de 2013).

19 Le travail sur le passé consiste à la découverte des crues dans l'histoire de la vallée au travers des documents d'archives (textes, images) et à mesurer leur inscription dans la mémoire familiale et collective (enquête auprès des familles, des élus et des responsables locaux menée par les élèves). Ces travaux viennent renforcer les connaissances des élèves sur les risques et sur la vulnérabilité des sociétés de leur vallée. Ils visent à les faire réfléchir sur la capacité des sociétés à se protéger.

Puis, le travail sur le(s) futur(s) est une phase d'initiation à la prospective à travers le témoignage d'acteurs locaux en charge de la prévention des risques et par la construction de maquettes par les élèves leur permettant d'exprimer un scénario d'aménagement du territoire afin que les habitants puissent vivre plus en sécurité. Cette étape prend notamment appui sur les programmes scolaires actuels de l'école élémentaire, qui stipulent explicitement pour le cycle 2 un travail de la compétence à "se repérer dans son environnement proche" (Bulletin officiel de l'Éducation nationale, $2015, \mathrm{n}^{\circ}$ spécial 11, p. 73 ) et pour le cycle 3 « une sensibilisation des élèves à la prospective territoriale» (ibid., p. 180) en lien avec les enjeux de l'« habiter ». Cette introduction des enjeux relatifs au futur constitue une nouveauté dans les programmes scolaires.

21 Enfin, les différentes productions des élèves réalisées tout au long de la séquence sont valorisées avec la confection d'une hypercarte. Cette dernière est une image numérisée sur laquelle sont créées des zones cliquables qui activent des documents ressources, ce qui favorise la lecture de la complexité du paysage et la mise en évidence de l'invisible comme les jeux d'acteurs (Partoune, et al., 2005). Ce document numérique sert de médium entre les classes et les parents. En effet, les élèves présentent leurs travaux à leurs proches ce qui permet de remobiliser et de diffuser leur réflexion sur les risques. 


\section{La maquette : repenser son territoire, sa vulnérabilité et sa possible résilience} risques de votre territoire, imaginer la vallée du Bastan en $2050 »$. Un temps de réflexion est accordé à chaque groupe de deux à trois élèves pour réfléchir aux solutions, ce qui, pour les deux classes, représente 11 groupes d'élèves. Chaque groupe a à sa disposition une photographie aérienne de la vallée au format A3 sur laquelle les villages ont été masqués par un fond vert identique aux prairies, ce qui limite l'influence de l'organisation spatiale actuelle (figure 4); une légende sous forme de tableau à compléter pour y intégrer les éléments de leur choix (figure 1); un sac avec du matériel pour modéliser les éléments du territoire (legos, ficelle, ruban, cailloux, pâte à modeler, papier de différentes couleurs). Les élèves placent à leur convenance sur la photographie aérienne: les maisons, l'école, la mairie, l'église, l'hôtel, le camping, les commerces, les voies de communication, la végétation, les éléments naturels, les aménagements et tout ce qu'ils souhaitent mettre dans leur maquette de la vallée.

Figure 1. Légende de la maquette d'élèves de cycle 2

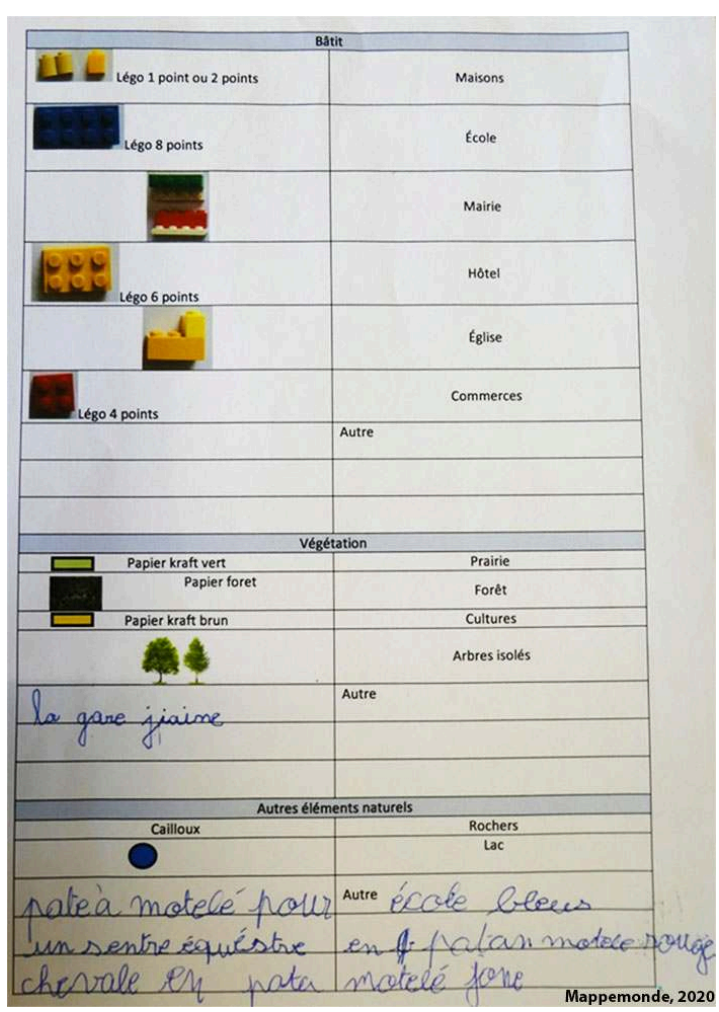

Une fois la maquette terminée, chaque groupe présente son travail à la classe puis les élèves sont questionnés par les enseignants et leurs camarades (demande d'éclaircissement, discussion des propositions, etc.). La figure 2 illustre cette phase : une élève de CM2 présente sa maquette, elle pointe les différents éléments, ici un hôtel (lego bleu), ce qui suscite l'intervention d'une camarade. Enfin une réflexion en commun est menée sur l'ensemble des projets proposés pour discuter de leur faisabilité, des acteurs à mobiliser et de la complexité de l'aménagement du territoire en fonction des risques pris en compte. 
Figure 2. Présentation d'une élève de $\mathrm{CM} 2$ avec pointage

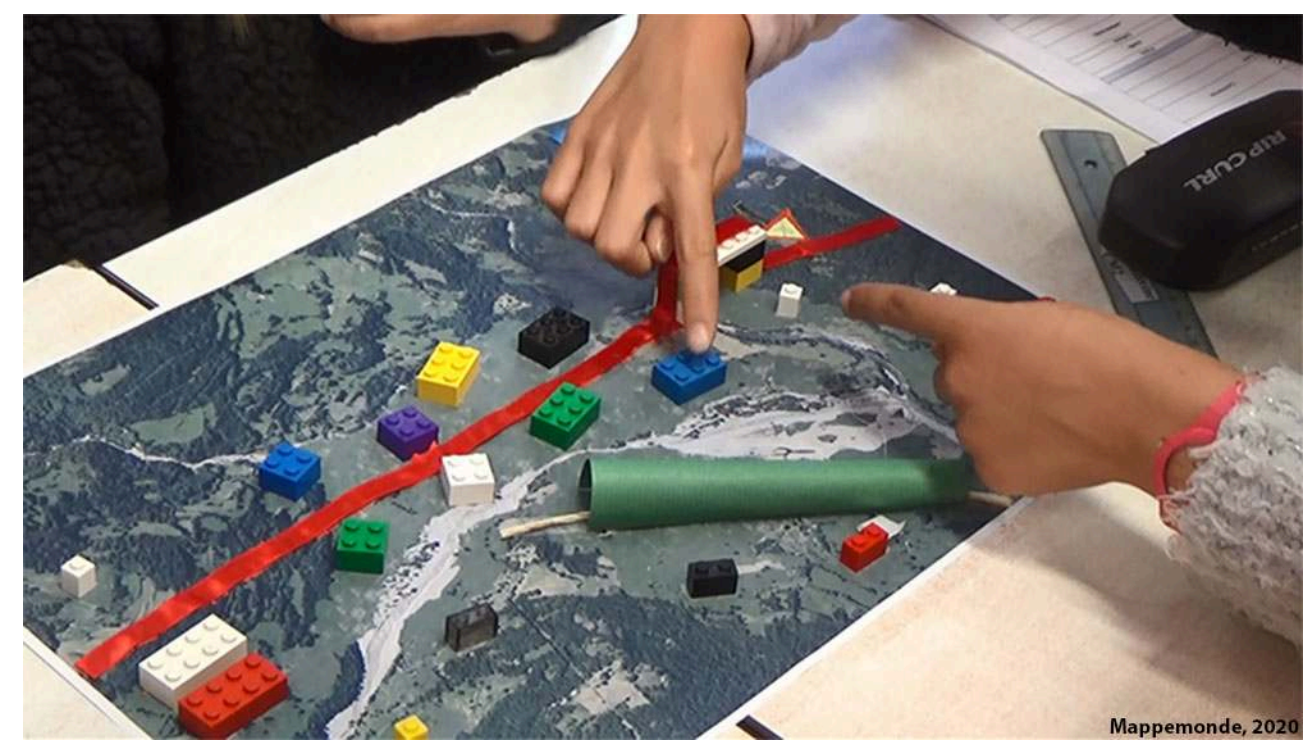

Le travail ainsi conduit visait à s'emparer d'éléments novateurs dans les attendus scolaires et de tester un dispositif pour en apprécier la faisabilité. Durant la mise en œuvre du dispositif, les chercheurs ont travaillé en étroite interaction avec les enseignants des classes concernées sur le mode de recherches collaboratives et ont pris part aux activités. Leur posture était donc celle de chercheurs impliqués. Néanmoins, les données recueillies lors de ces activités ont permis a posteriori à l'équipe d'engager un regard objectivant sur le travail effectif des élèves et d'apprécier l'efficacité du dispositif au regard des objectifs fixés.

\section{Recueil et analyse des corpus}

Les données analysées dans cet article relèvent de deux corpus de nature différente: d'une part les maquettes et leur légende sous forme de tableau, d'autre part la présentation orale des maquettes et les échanges à leur sujet.

Chaque maquette, avec sa légende, est analysée du point de vue des éléments représentés, de leur nombre, de leur localisation, et des relations entre ces éléments. Cette première analyse qualitative et descriptive donne lieu à l'établissement des types de risques mobilisés par les élèves, de l'évaluation du degré de changement par rapport au territoire actuel et d'un critère de cohérence entre les propositions d'aménagement et la prise en compte des risques dans chacun des groupes. Le contenu des onze maquettes peut ainsi être comparé entre groupes du cycle 2 et du cycle 3 ainsi qu'entre élèves de même cycle.

Ensuite, chaque présentation orale est analysée d'un point de vue quantitatif et qualitatif à l'aide d'une typologie des questions (quoi, où, pourquoi, comment, et si ?) et des réponses (oui-non, pas de réponse, nommer/localiser/décrire, explication et justification, modification du point de vue). Les pointages sont aussi comptabilisés (figure 2). Cette analyse permet notamment de comparer les discours des élèves de cycle 2 et de cycle 3 , de questionner leurs propositions et d'observer les arguments avancés. La remobilisation éventuelle d'éléments rencontrés dans les différentes 
temporalités lors de la séquence pédagogique est observée dans leurs propositions d'aménagement du territoire.

\section{Résultats : analyse des maquettes et de leur présentation par les élèves}

Les formats de présentation sont globalement équivalents entre les groupes. Nous ne notons pas de différences entre cycle 2 et 3 sur la durée totale ni sur le nombre moyen d'interactions et le nombre moyen de réponses fournies par le groupe lors de la présentation de sa maquette (tableau 1).

Tableau 1. Durée, nombre d'interactions et nombre de réponses pour les groupes du cycle 2 et du cycle 3

\begin{tabular}{|l|l|l|l|}
\hline & Durée totale (sec.) & Nombre d'interactions & Nombre de réponses \\
\hline Cycle 2 & $482 \pm 166$ & $109 \pm 29$ & $36 \pm 8$ \\
\hline Cycle 3 & $510 \pm 166$ & $107 \pm 47$ & $41 \pm 18$ \\
\hline Total & $497 \pm 158$ & $108 \pm 38$ & $39 \pm 14$ \\
\hline
\end{tabular}

En revanche, l'analyse qualitative des maquettes (et de leur présentation), ainsi que des réponses apportées par les groupes aux questions par les pairs ou les enseignants, permet d'identifier différentes postures chez les élèves.

\section{Analyse des maquettes}

Types de risques pris en compte dans les projets

Les crues et inondations représentent le type de risque majoritairement pris en compte dans les propositions des élèves puisqu'elles sont figurées dans neuf légendes de maquettes sur les onze produites (tableau 2). Ensuite, les éboulements ou glissements de terrain constituent le deuxième risque le plus cité. Les avalanches sont présentes dans cinq légendes de maquettes. Deux propositions intègrent le risque lié aux incendies, une autre celui lié aux séismes et enfin une autre celui lié au passage des voitures. Chaque groupe prend en compte en moyenne 2,2 risques.

Tableau 2. Types de risque présents dans la légende de la maquette pour chaque groupe

\begin{tabular}{|l|l|l|l|}
\hline Types de risque & Cycle 2 $(\mathrm{N}=5)$ & Cycle 3 (N = 6) & Total (N = 11) \\
\hline Crues et inondations & 5 & 4 & 9 \\
\hline Éboulements et glissements de terrain & 2 & 4 & 6 \\
\hline Avalanches & 4 & 1 & 5 \\
\hline
\end{tabular}




\begin{tabular}{|l|l|l|l|}
\hline Incendies & 0 & 2 & 2 \\
\hline Séismes & 0 & 1 & 1 \\
\hline Voitures (se faire écraser) & 0 & 1 & 1 \\
\hline
\end{tabular}

31 Si les crues et inondations constituent le premier risque pour les élèves des deux cycles, ce sont les avalanches qui interviennent en deuxième position pour le cycle 2 et les éboulements pour les cycles 3 .

\section{Degré de changement dans les projets des élèves}

À partir de l'analyse des bâtiments présents sur les différentes maquettes et de l'écart avec la situation actuelle, nous avons catégorisé les propositions des élèves selon trois degrés de changement (figure 3). Il apparait qu'un seul groupe propose une reproduction de la situation actuelle, tant par la nature des bâtiments similaire à l'existant que par leur localisation sur la carte. À l'opposé, quatre groupes effectuent des changements partiels par rapport à la situation actuelle, on note dans leurs propositions des déplacements de bâtiments associés à une permanence de certains éléments. Par exemple, un groupe du cycle 2 qui a pourtant pris en compte le risque lié aux crues laisse une école positionnée au bord du Gave alors qu'il en déplace d'autres. Enfin, les six autres groupes témoignent dans leur projet d'aménagement d'une recomposition plus aboutie du territoire. Il s'agit dans ce cas d'une reconfiguration de l'aménagement du territoire, avec déplacement et création de bâtiments ainsi que des voies de communication (routes, chemin de fer...).

Figure 3. Degré de changement dans les projets présentés

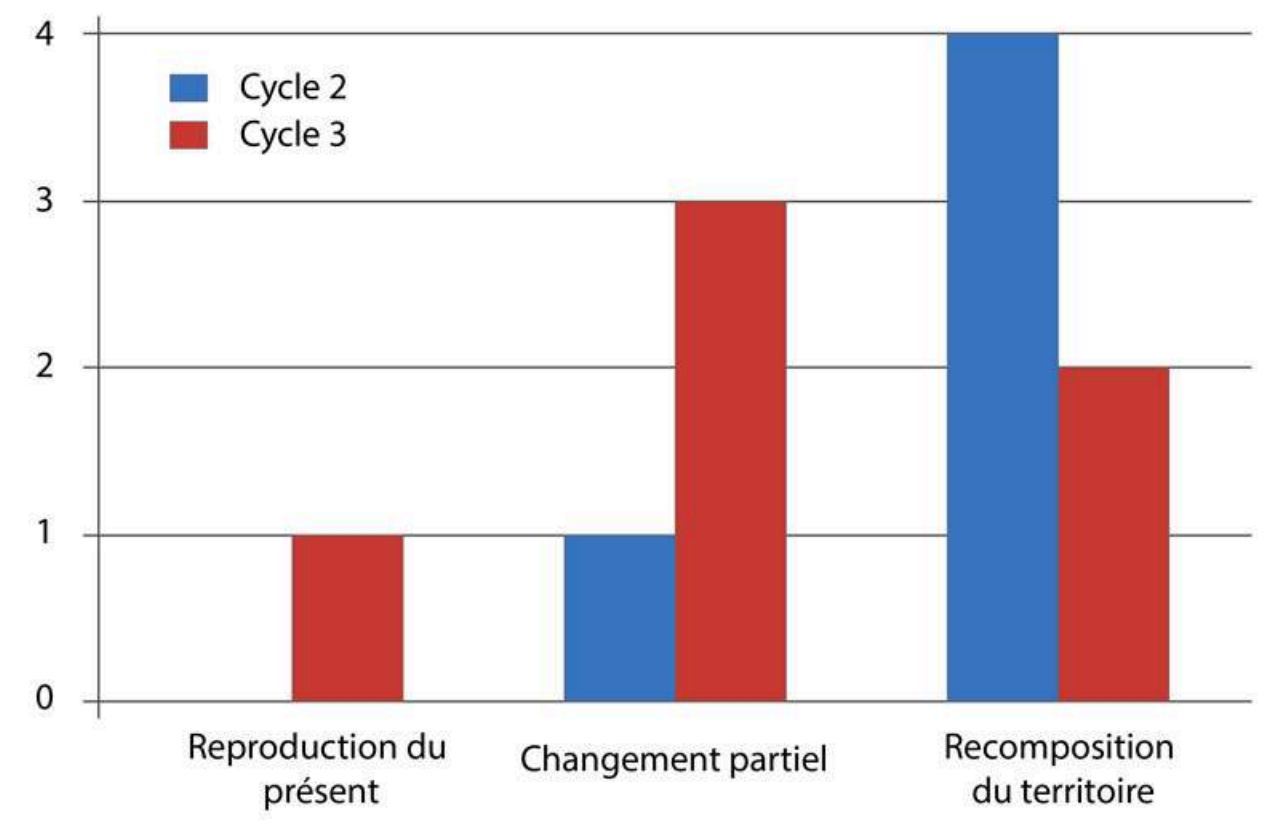

Mappemonde, 2020 
Cette analyse rend compte d'un changement plus prononcé dans les propositions des élèves du cycle 2 par rapport à celles des élèves du cycle 3 . Les solutions techniques et les aménagements proposés, notamment par rapport aux crues, consistent à construire des barrages, des digues de protection, à élargir le lit de la rivière avec une reconstruction des routes sur les hauteurs (figure 4), ou à aménager des zones «tampons». Ces travaux envisagés doivent aider l'eau à mieux s'écouler ou à retenir l'eau en amont et ils s'accompagnent d'un déplacement de l'habitat loin des cours d'eau. Par ailleurs, certaines propositions font preuve de réponses différenciées selon la nature des bâtiments. En effet, si les habitations sont pour la plupart déplacées loin des berges, ce n'est pas le cas des commerces. Les élèves le justifient par rapport à la nature du bâtiment : «ça c'est pas grave, c'est des commerces ».

Figure 4. Maquette d'élèves de cycle 3

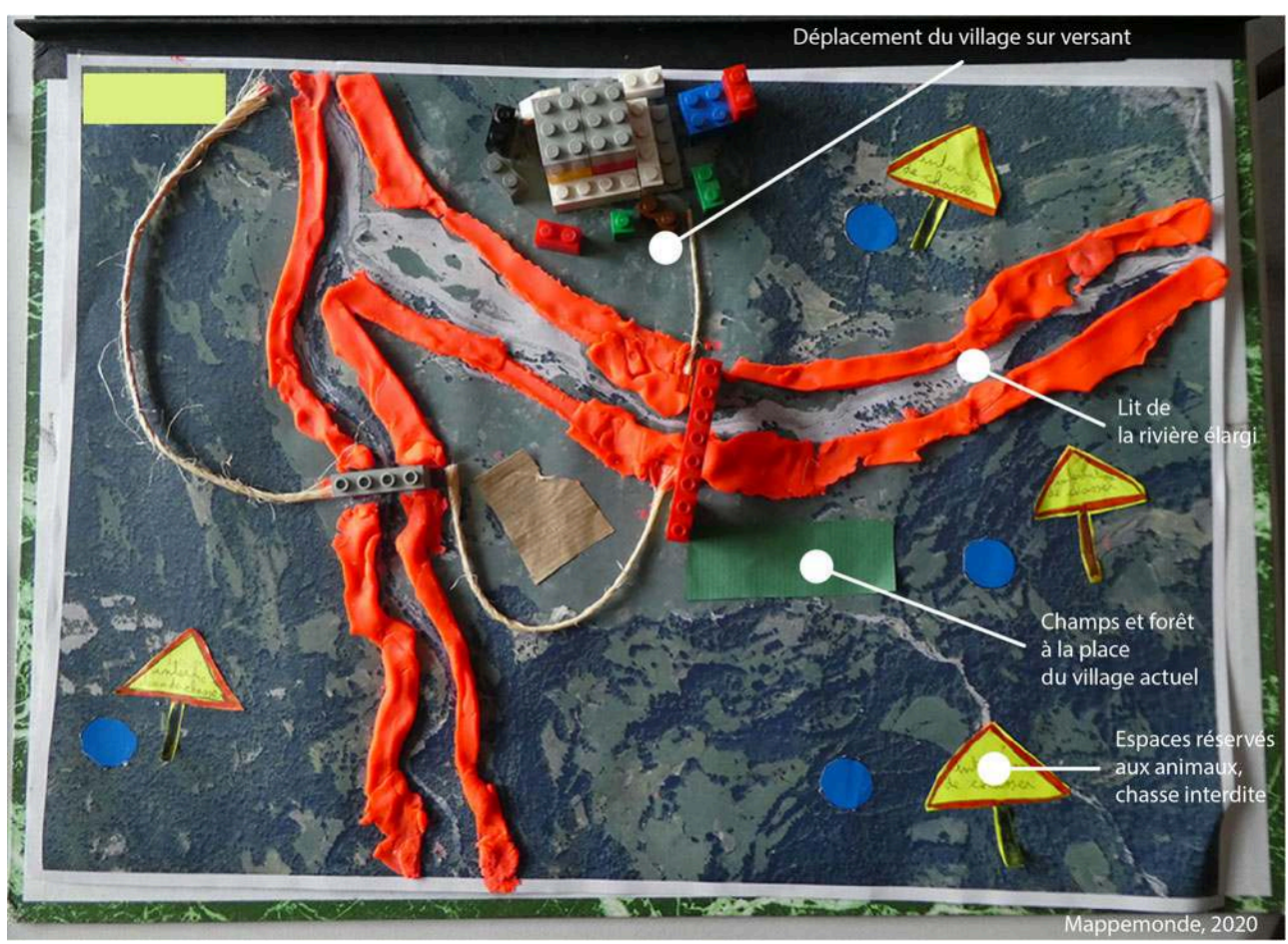

Les maquettes peuvent également révéler de nouvelles formes d'habiter, avec une organisation sectorielle (figure 5) : maisons regroupées à un endroit, commerces à un autre, écoles encore ailleurs et gare en dehors du village. 
Figure 5. Maquette d'élèves de cycle 3

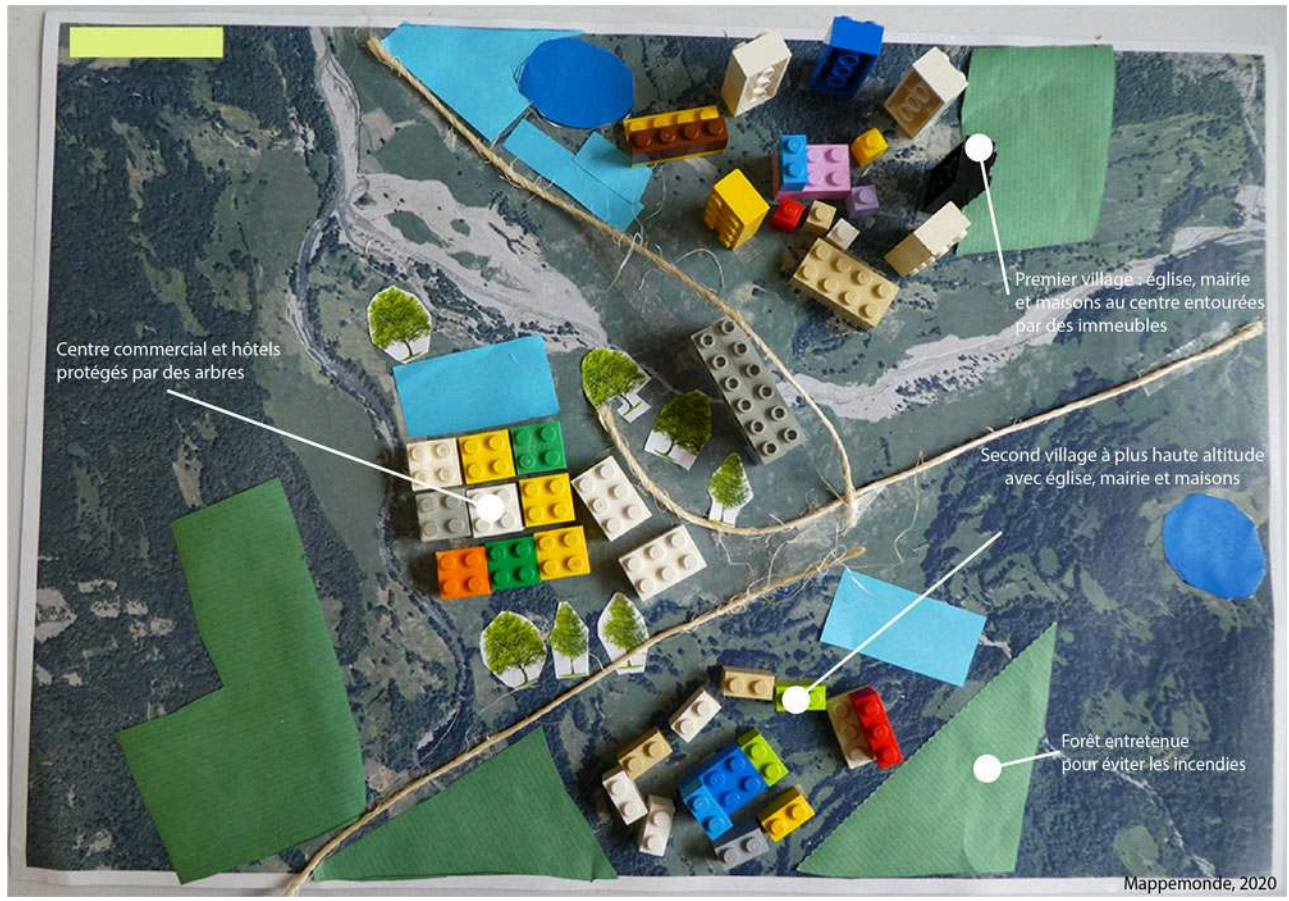

Certains élèves optent au contraire pour un regroupement de l'ensemble du bâti et même parfois de manière originale au sein d'une fortification (figure 6). Cette organisation s'appuie aussi sur de nouveaux modes de déplacements avec l'intégration de voies ferrées et d'ouvrages d'art (pont, tunnel).

Figure 6. Maquette d'élèves de CM

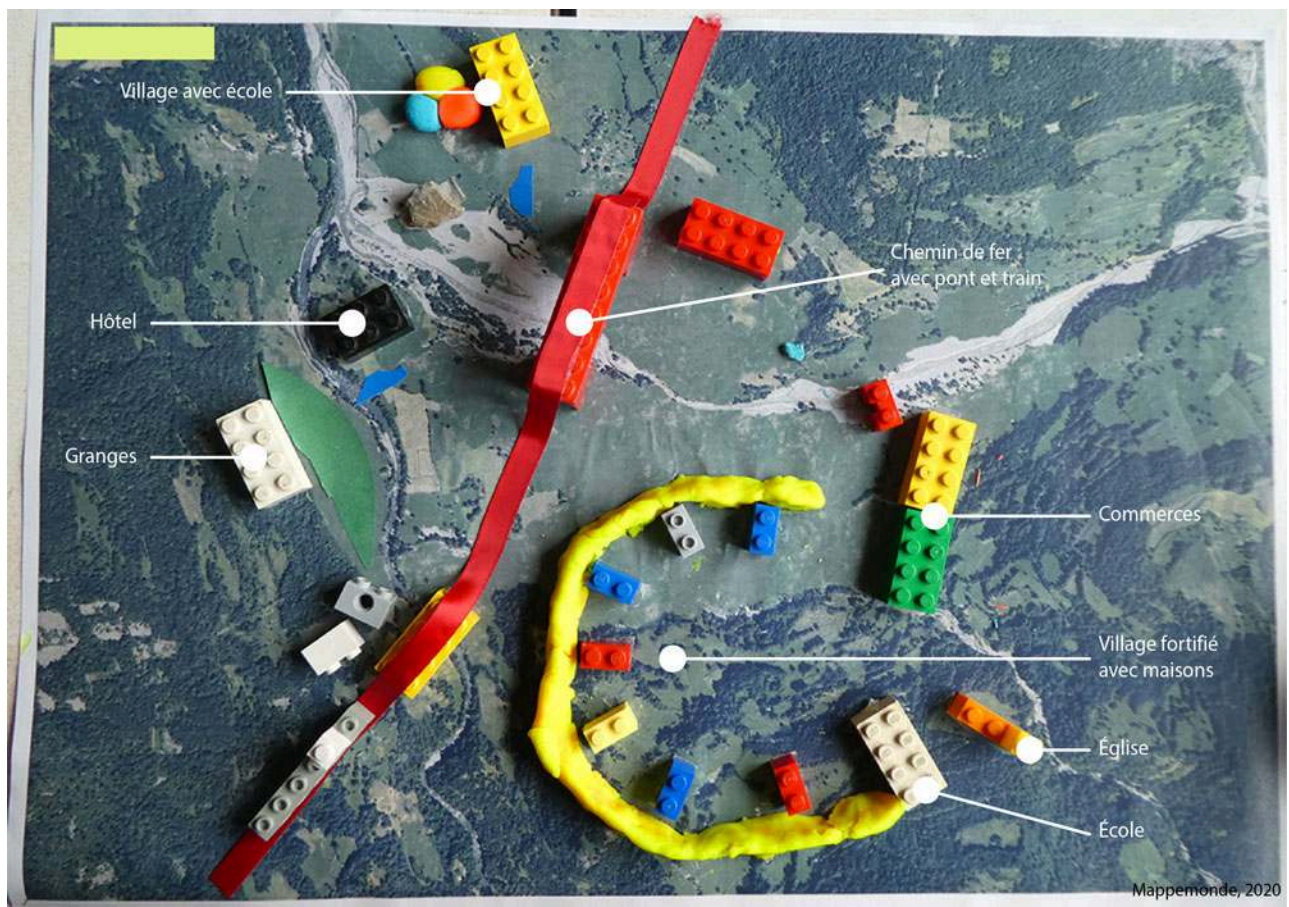




\section{Analyse des réponses apportées par chaque groupe aux questions posées sur son projet}

\section{Typologie des réponses}

L'analyse des présentations des maquettes a permis de distinguer cinq types de réponses (tableau 3).

Tableau 3. Typologie des discours lors de la présentation de la maquette par chacun des groupes

\begin{tabular}{|l|}
\hline Typologie des discours \\
\hline R1 : pas de réponse ou « je ne sais pas » \\
\hline R2 : réponse binaire oui ou non \\
\hline R3 : nommer, localiser, décrire \\
\hline R4 : explication, justification, développement de son point de vue (parce que, pour que) \\
\hline R5 : modifie son point de vue
\end{tabular}

Il n'y a pas de différences notables entre les deux cycles (figure 7): les réponses apportées par les groupes concernent principalement des indications pour nommer, localiser et décrire les éléments présents sur la maquette (R3) ainsi que des dimensions explicatives sur ces éléments et leurs relations entre eux (R4). Ce résultat est cohérent avec l'objectif de la présentation et la consigne donnée aux élèves. La rareté de la modification de point de vue (R5) s'explique de la même manière et elle s'observe lors des discussions et débats dans les groupes d'élèves les plus âgés. 
Figure 7. Répartition des types de réponses en \% pour les 5 groupes du cycle 2 et les 6 groupes du cycle 3

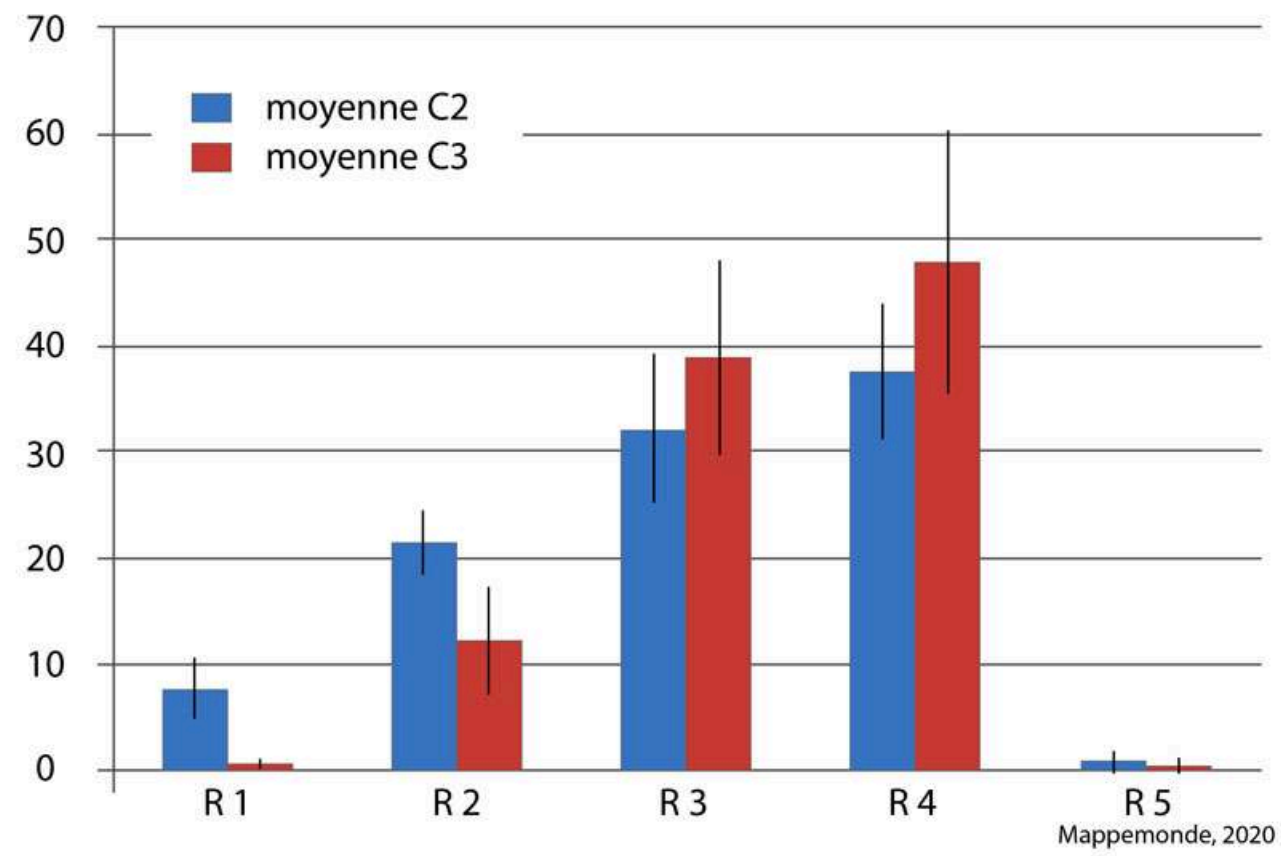

Cette répartition se retrouve lorsque l'on analyse les types de réponses par groupe. Malgré des variations entre groupes, les localisations et descriptions (R3) et les explications (R4) des élèves représentent pour chaque groupe la plus grande proportion de réponses (figure 8).

Figure 8. Proportion des types de réponses par groupe (\%). Les groupes 1 à 5 correspondent aux élèves de cycle 2 et les groupes 6 à 11 aux élèves de cycle 3

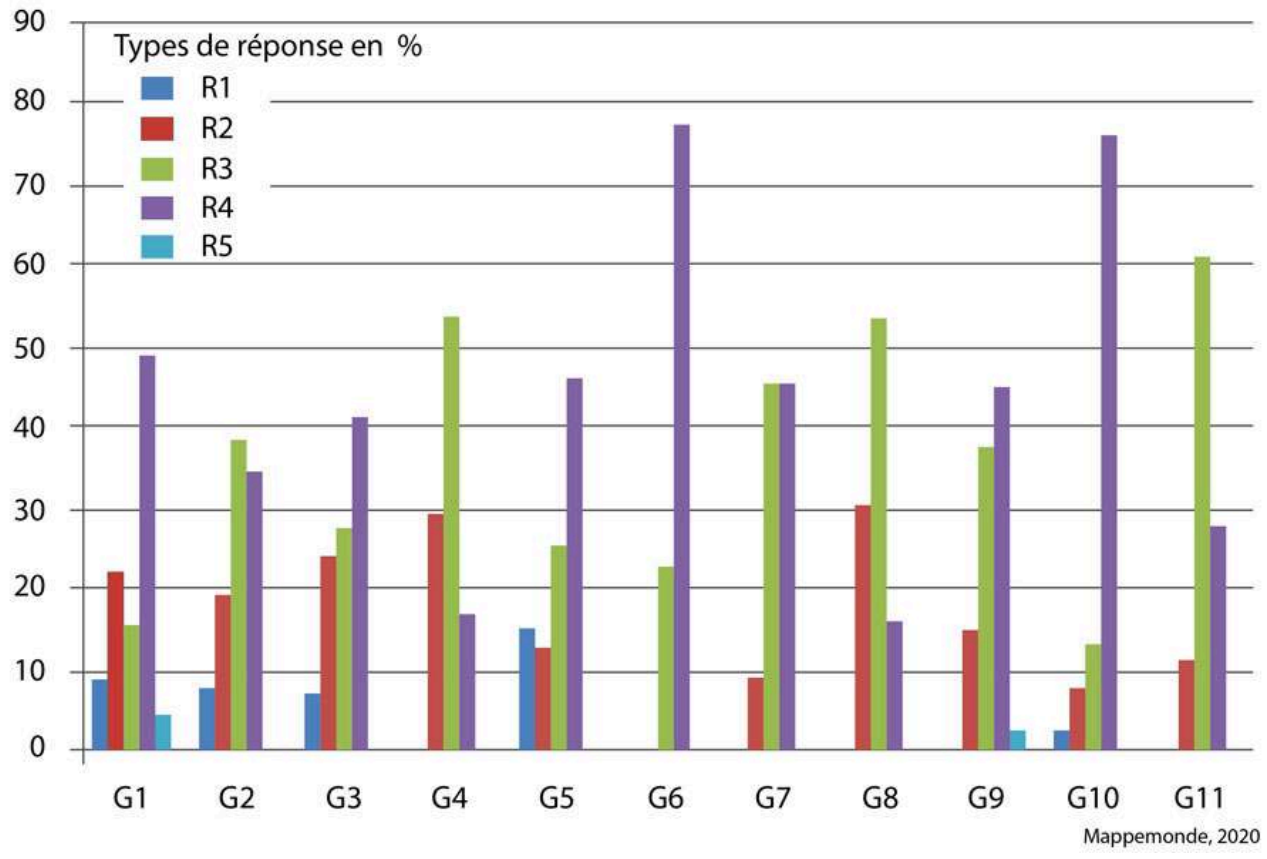




\section{Proportion des réponses avec pointage sur la maquette}

Les pointages lors de la présentation de la maquette sont utilisés en moyenne pour près de moitié des réponses de chaque groupe $(49 \% \pm 15)$. La variation entre groupes est importante (figure 9), puisque le groupe utilisant le moins le pointage l'effectue pour un peu plus du quart des réponses (groupe 7) alors que le groupe qui en propose le plus (groupe 9) l'utilise pour pratiquement $3 / 4$ de leurs réponses.

Figure 9. Pourcentage des pointages sur le nombre total de réponses par groupe

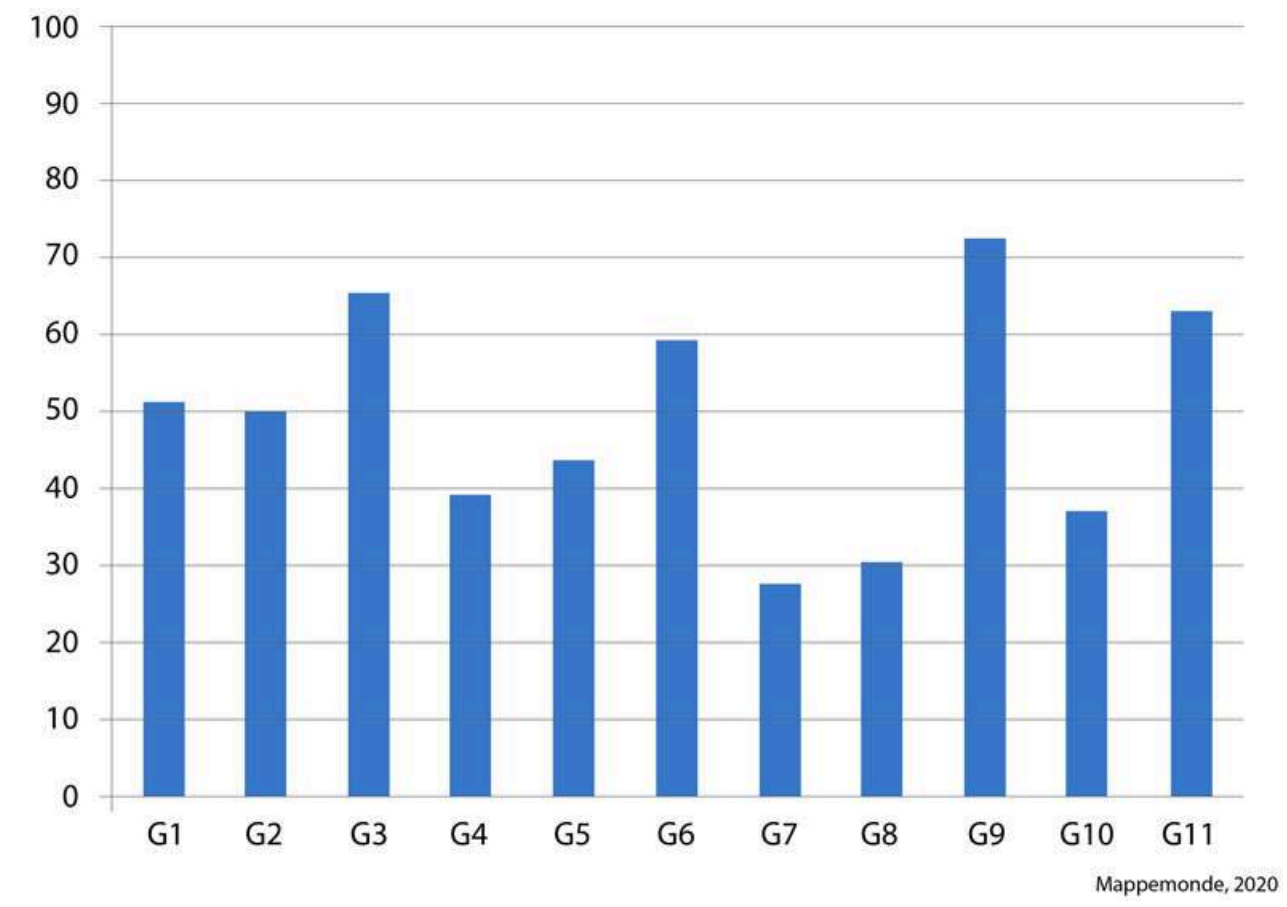

Groupes 1 à 5 en cycle 2 et 6 à 11 en cycle 3.

Malgré cette différence de fréquence, le pointage a souvent la même fonction (figure 10) : il accompagne la description, la localisation (R3) et l'explication (R4) que les élèves soient en cycle 2 ou en cycle 3. 
Figure 10. Répartition des pointages selon la nature de la réponse

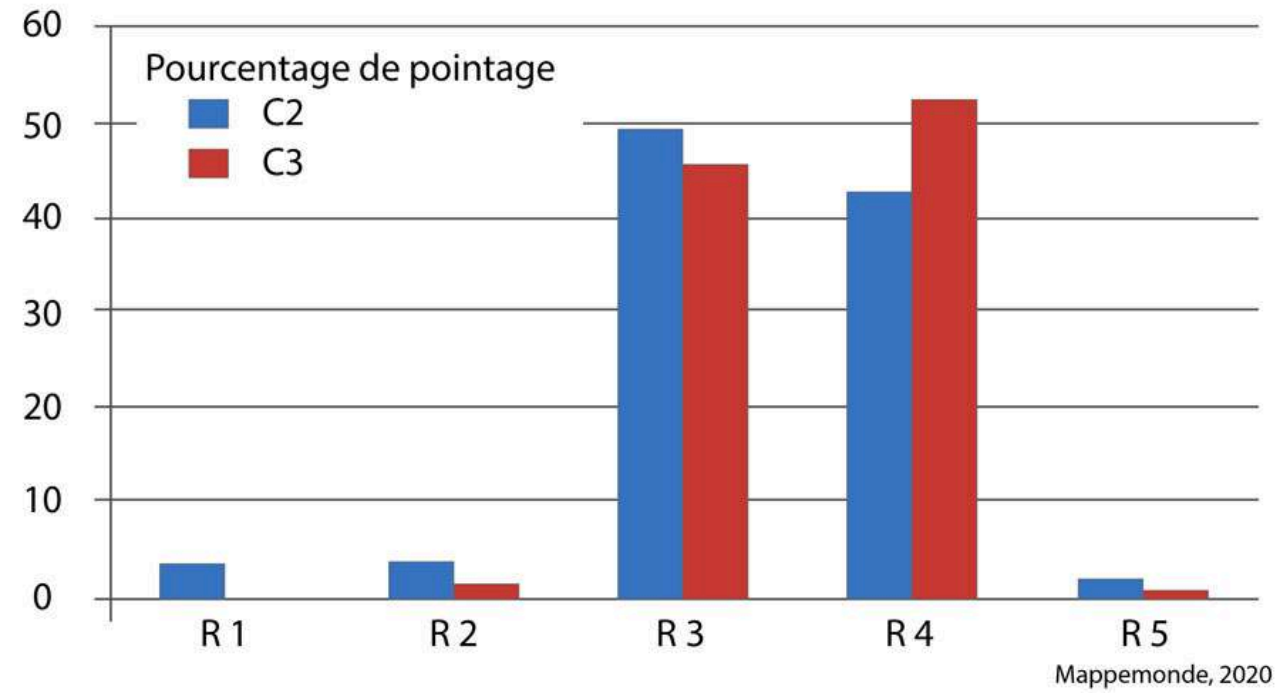

Enfin, nous avons également distingué les pointages sur des éléments en deux dimensions (pointages «2D») des pointages qui intègrent, dans les propos qui les accompagnent, des dimensions de hauteur (sous, par-dessus, par-dessous...). Ces derniers sont qualifiés de "3D» puisqu'ils intègrent 3 dimensions de l'espace représenté. Ils sont minoritaires et représentent selon les groupes de 0 à $40 \%$ des pointages, et en moyenne autour de $14 \%$ des pointages avec une forte disparité entre groupes (écart type \pm 14 ).

\section{La maquette : un outil de réflexion spatiale et citoyenne}

\section{La dimension 3D des maquettes, un apprentissage de l'espace}

Le support donné aux élèves pour la construction de leur projet sous forme de maquette était une photographie aérienne verticale en couleur du territoire d'Esquièze et ses environs, soit une représentation zénithale en deux dimensions. Ce «fond de maquette » n'a cependant pas posé de problème aux élèves dans la mesure où l'espace représenté leur est bien connu et a été travaillé pendant la première partie du projet. Ainsi, le relief est globalement bien pris en compte dans les propositions des élèves et les explicitations qu'ils en donnent, notamment sous l'angle de la dénivellation et de la segmentation du lieu par des cours d'eau d'inégale importance (Gave, Bastan).

Sur ce fond, les réalisations ont mobilisé deux modalités d'expression qu'offre une maquette: la hauteur des éléments et leur superposition éventuelle. La hauteur des maisons et édifices commerciaux ou publics est le levier le plus mobilisé, par l'utilisation de legos de tailles différentes. Les élèves ont remobilisé avec facilité les exemples donnés dans la légende. Dans la majorité des cas, elle permet seulement de différencier les types d'espaces bâtis sous l'angle de l'organisation spatiale (quartiers, types de villages). Cependant, pour certains groupes de cycle 3 , son usage va plus loin dans la mesure où la hauteur des bâtiments est associée à des enjeux de protection par rapport au risque d'inondation. Pour un de ces groupes, des maisons sur pilotis ont 
même été réalisées dans la zone la plus exposée aux crues. La superposition est également mobilisée notamment par la représentation de ponts permettant le franchissement des cours d'eau ou le croisement d'axes de transport (voie ferrée, routes) (figures 4, 6, 11). Des tunnels sont aussi envisagés par quelques groupes (figure 11), pour faciliter les franchissements (passer sous la rivière) ou pour protéger les axes des chutes de blocs. Difficiles à réaliser sur un fond de maquette en 2D, ils ont été matérialisés par des sortes de tranchées couvertes ou simplement dits à l'oral.

Figure 11. Maquette d'élèves de cycle 3

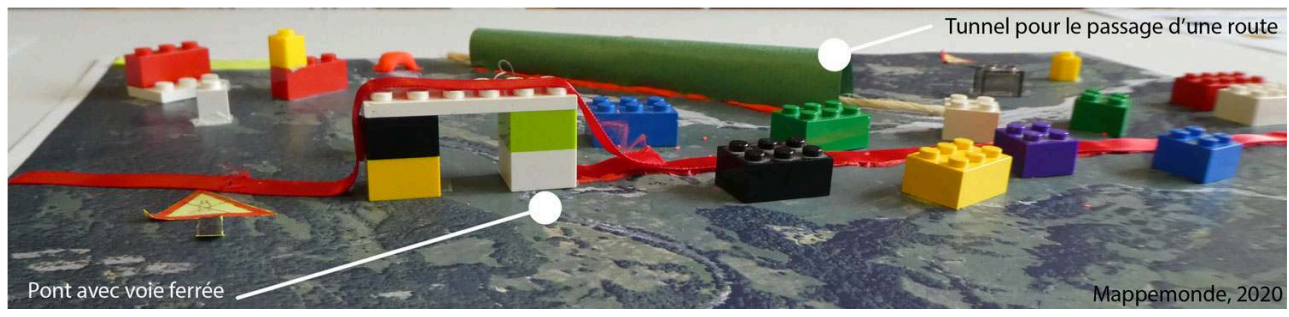

Les présentations orales des maquettes ont par ailleurs permis d'apprécier plus finement le rôle de la 3D qu'apporte la maquette. Dans la présentation de leur maquette, les élèves ont mobilisé un vocabulaire de situation : " en hauteur ", "plus en hauteur", "plus haut», "très haut», "par-dessus", «sous terre», etc. Ces expressions sont souvent accompagnées de gestes indiquant les hauteurs relatives des lieux pointés en appui à leur discours. La dimension 3D déjà présente par les éléments de la maquette s'en trouve ainsi amplifiée et surtout intègre les dénivellations topographiques que le fond de maquette ne rend pas visibles. Dans certaines interactions entre les auteurs des maquettes et les autres élèves, la 3D est aussi présente, soit par des questions sur les hauteurs des bâtiments ou la situation des éléments (bâti, axes, lac, forêt, rochers...) le long des versants visant à mieux comprendre la maquette soit par des questionnements et remarques sur la fonctionnalité des superpositions.

\section{Prise de conscience des risques et réflexion sur les aménagements pour diminuer la vulnérabilité}

Les maquettes témoignent d'une prise de conscience par les élèves des risques présents sur leur territoire. En effet, au départ de notre expérimentation, les élèves minoraient les crues/inondations, alors que les avalanches, les éboulements et les accidents du quotidien étaient au cœur de leurs discours. Les projets de maquette ont majoritairement été centrés sur les inondations, les élèves se sont donc approprié la séquence qui ciblait essentiellement cet aléa. Il semble ainsi que les élèves aient pris conscience de l'importance de ce risque sur leur territoire, ce qui constitue un des premiers objectifs d'une éducation aux risques ${ }^{4}$. Cela se manifeste par la mise en place d'infrastructures de protection : barrage, digue, mur en fer, enrochement (figure 12); et aussi par le déplacement des bâtiments sur des zones moins exposées. Ainsi, les villages actuels sont souvent déplacés plus loin du lit de la rivière et reconstruits sur les pentes de la montagne (figures 4, 5, 6, 12). 
Figure 12. Maquette d'élèves de cycle 2

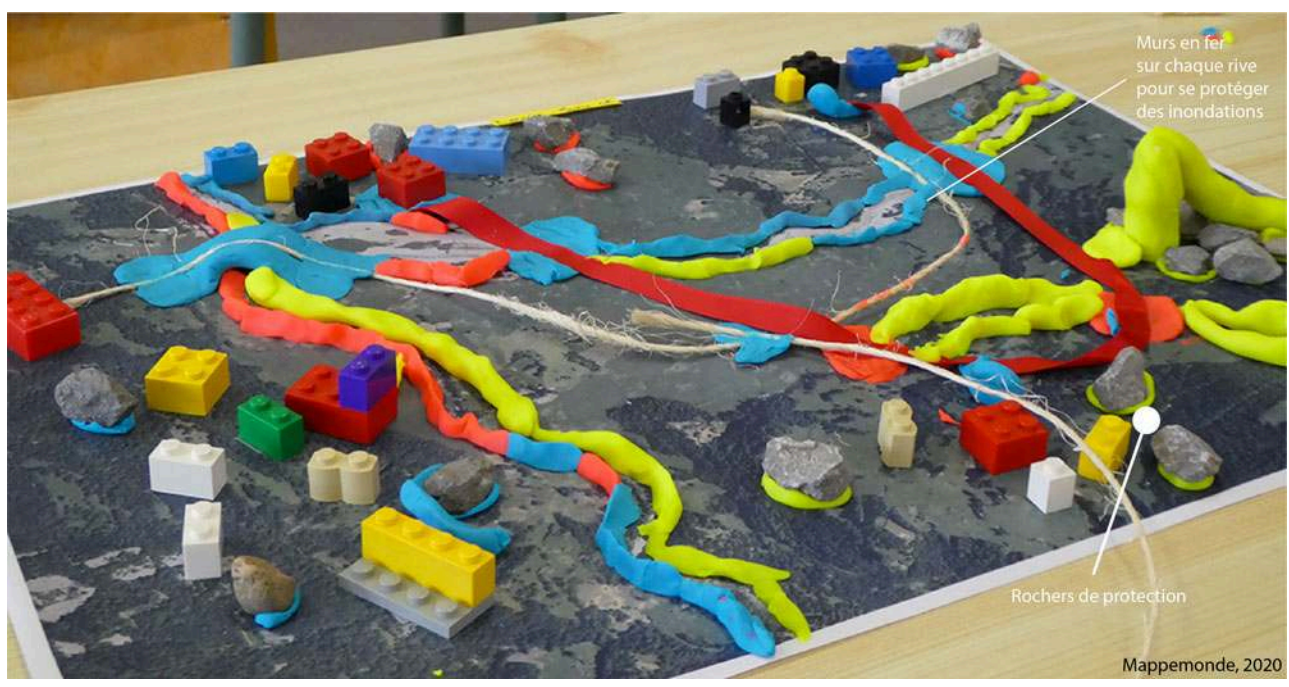

Il semblerait donc que les élèves pensent leur territoire à travers leur vulnérabilité : les zones inondables sont abandonnées (figures $4,6,12$ ) pour « laisser l'eau passer », elles sont transformées en prés et en champs.

De nouveaux types d'habitats sont aussi envisagés et les élèves font preuve d'innovations en mobilisant des aménagements présents sur d'autres territoires: immeubles qui font office de mur de protection contre les crues, maisons et routes sur pilotis, bâtiments à plusieurs étages pour éviter l'étalement et les inondations (figure 5 ) ou, au contraire, maisons basses pour amoindrir les dégâts lors des tremblements de terre. Nous observons une spécialisation de l'espace avec des plantations de forêt pour se protéger des éboulements ou dans un but de préservation de la nature (figure 4), des zones d'habitation, des zones de cultures ou d'activités, le tout étant connecté par des voies de communication qui se veulent parfois plus écologiques (train, voie pour voitures électriques). Il faut remarquer que les élèves de cycle 3 ont de manière inconsciente cherché à diminuer la présence des voitures qui étaient source d'accidents et vues comme un risque au début de l'expérimentation. Ils ont ainsi construit des parkings, des voies ferrées et des gares.

Ainsi, nous constatons une forte cohérence des projets d'aménagement, car sept projets sur onze adaptent correctement leurs réponses aux risques envisagés. Les élèves remobilisent convenablement des connaissances apportées lors de la séquence sur les risques (notamment par le chercheur et l'adjoint au maire responsable de la sécurité et des risques). Par conséquent, cette éducation aux risques aboutit à une hybridation des savoirs, soit un processus d'articulation de savoirs qui permet d'approcher la complexité (Bédouret et al., 2018) dans laquelle les élèves utilisent leurs propres connaissances du terrain et celles apportées par les différents acteurs du projet pédagogique.

La maquette a été aussi un support favorisant la réflexion et le débat autour des solutions possibles face aux risques. Chaque groupe a présenté et expliqué ses choix puis les camarades ont pu poser des questions et faire des commentaires. Les élèves ont dû se justifier et chercher des arguments. Ce moment a permis une réflexion autour des incohérences des localisations des habitations ou des voies de communication, ainsi le tunnel où passe la route (figure 11) a été remis en cause par rapport au risque de 
tremblement de terre, ou la localisation du village fortifié trop proche d'un torrent (figure 6) est dénoncée. Cette phase est suivie d'une discussion collective où un certain nombre de questions sont évoquées : le projet le plus réalisable, faisabilité et coût des projets, rôle des acteurs, etc.

Par conséquent, la maquette «sert de fond spatial à la discussion des différentes idées explicatives " (Orange et al., 2001). Les échanges à l'oral vont eux permettre de rendre dynamique ce modèle par les explications apportées par les élèves. Comme dans les débats explicatifs analysés dans l'étude d'Orange et al., (2001), la maquette (comme le ferait un écrit de type schéma) permet de fixer provisoirement les propositions des élèves, et fournit alors un support concret à la discussion. Toutefois, elle va au-delà du " fond spatial », car elle permet de réellement prendre en compte la dimension spatiale des risques. Par-là, elle nourrit les représentations des élèves et enrichit leur capital spatial (Lévy, 2019, p. 149).

51 Ce temps du travail permet donc l'acquisition de compétences sociales (écoute de l'autre, respect des points de vue, capacité à argumenter) et positionne les élèves dans une posture active de citoyen conscient. Ainsi, l'éducation aux risques s'intègre de manière plus globale à l'éducation au développement durable dont un des objectifs est de mettre en dialogue les savoirs des acteurs et de faire émerger une véritable intelligence citoyenne (Julien et al., 2014 ; Girault et al., 2008).

\section{Conclusion}

52 La construction de maquettes met les élèves dans un travail de prospective basé sur une réflexion complexe et systémique où la question de l'habiter est interrogée (se loger, travailler, se déplacer, se divertir) et où la relation société-nature est analysée à travers les risques. Dans les conditions de l'expérimentation, l'élaboration de maquettes de leur territoire favorise, pour les élèves, une prise de conscience des vulnérabilités et une meilleure connaissance des aléas et des risques. En proposant des aménagements en cohérence avec les risques de leur territoire et les arguments d'acteurs locaux, ils témoignent d'une intégration de savoirs multiréférencés, d'une articulation de savoirs d'origines diverses.

53 L'élaboration de maquettes contribue ainsi à une éducation aux risques en renforçant une culture du risque (prévention, connaissance du danger et des gestes à accomplir) et en développant une réflexion sur les solutions pour rendre la société plus résiliente. Ce dispositif, ponctué de nombreuses phases de confrontations collectives et d'échanges, montre qu'au sein d'un territoire de multiples points de vue coexistent et s'entrechoquent, rendant possibles des moments d'apprentissage démocratique. 


\section{BIBLIOGRAPHIE}

ANTOINE J.-M. (2010). Géohistoire des catastrophes et des risques torrentiels. Une histoire de l'environnement pyrénéen. HDR, Géographie. Université Toulouse le Mirail - Toulouse II.

BARTHES A., BLANC-MAXIMIN S., DORIER-APPRIL E. (2019). « Quelles balises curriculaires en éducation à la prospective territoriale durable? Valeurs d'émancipation et finalités d'implications politiques des jeunes dans les études de cas en géographie ». Éducation et socialisation, $\mathrm{n}^{\circ}$ 51. En ligne : http:// journals.openedition.org/edso/5755; DOI : 10.4000/edso.5755

BÉDOURET D., VERGNOLLE MAINAR C., CHALMEAU R., JULIEN M. P., LÉNA J. Y. (2018). « L'hybridation des savoirs pour travailler (sur) le paysage en éducation au développement durable ». Projets de paysage. En ligne : http://www.projetsdepaysage.fr/fr/

1_hybridation_des_savoirs_pour_travailler_sur_le_paysage_en_ducation_au_d_veloppement_durable. BIROU A. (dir.) (1966). Vocabulaire pratique des sciences sociales. Paris : Éditions ouvrières, 314 p. BLANC-MAXIMIN S., FLORO M. (2017). «L'éducation au territoire à l'école élémentaire : le cas des projets PNR Queyras-EN ». In BARTHES A., CHAMPOLLION P., ALPE Y. (dir.), Permanences et évolutions des relations complexes entre éducations et territoires, Londres : ISTE Éditions, p. 175-192.

BLESIUS J.-C. (2013). « Discours sur la culture du risque, entre approches négative et positive. Vers une éducation aux risques? ». Géographie et cultures, $n^{\circ} 8$. En ligne $:$ http:// journals.openedition.org/gc/3141; DOI : 10.4000/gc.3141

BODUR B., GUICHARD J. (2006). « Simuler un phénomène biologique, cas de la flexion-extension du bras ». Aster, n 43 , p. 35-56.

BRUNET R. et al. (1993). Les mots de la géographie. Montpellier/Paris : GIP RECLUS/La Documentation française.

BUYCK J., TIXIER N., RAMIREZ-COBO I. (2016). « La maquette in situ comme pédagogie immersive du projet urbain. Des étudiants au cœur du quartier des Escanaux à Bagnols-sur-Cèze. À quoi joue-ton? ». L'urbanisme, l'architecture et le jeu. Jouer pour mieux régner, avec ou sans l'ego ? École nationale supérieure d'architecture et de paysage de Lille, déc. 2016, Lille, France. En ligne : https:// hal.archives-ouvertes.fr/hal-01590591

CLERVEAUX V., SPENCE B. (2009). "The Communication of Disaster Information and Knowledge to Children Using Game Technique: The Disaster Awareness Game (DAG)". International Journal of Environmental Research, vol. 3, nº 2, p. 209-222. DOI : 10.22059/ijer.2010.48

CONSIDÈRE S., GRISELIN M. (1997). « La classe paysage ». Mappemonde, nº 97/3. En ligne : http:// www.mgm.fr/PUB/Mappemonde/M397/Considere.pdf

DROUIN A.-M. (1988). « Le modèle en question ». Aster, $\mathrm{n}^{\circ}$ 7, p. 1-20.

DUSSAUX M. (2017). « L'éducation au territoire ». In A. BARTHES, J.-M. LANGE et N. TUTIAUX GUILLON. Dictionnaire critique des enjeux et concepts des « éducations à ». Paris : Éditions L'Harmattan, 624 p. ISBN 978-2-343-12678-4

FERRAS R. (1993). Les modèles graphiques en géographie. Paris : Economica.

FRÉZAL-LEININGER C. (2014). « Une rhétorique des images du risque dans la géographie scolaire ». M@ppemonde, $\mathrm{n}^{\circ}$ 113. En ligne : https://mappemonde-archive.mgm.fr/num41/articles/ art14103.html 
GIORDAN A., VECCHI G. DE (1987). Les origines du savoir. Des conceptions des apprenants aux concepts scientifiques. Neuchâtel : Delachaux et Niestlé, 203 p.

GIRAULT Y., SAUVÉ L. (2008). « L'éducation scientifique, l'éducation à l'environnement, et l'éducation pour le développement durable». Aster, n 46, p. 7-30.

GODIN M.-A. (2008). « À la découverte de l'espace vécu, ou : commencer la géographie dès l'école maternelle ». Cahiers pédagogiques, $\mathrm{n}^{\circ} 460$, p. 38-40.

HOFFMANN R., MUTTARAK R. (2017). "Learn from the Past, Prepare for the Future: Impacts of Education and Experience on Disaster Preparedness in the Philippines and Thailand". World Development, $\mathrm{n}^{\circ}$ 96, p. 32-51. En ligne : https://doi.org/10.1016/j.worlddev.2017.02.016 HOST V. (1989). « Systèmes et modèles : quelques repères bibliographiques ». Aster, $\mathrm{n}^{\circ} 8$, p. 187-209.

JULIEN M.-P., CHALMEAU R., VERGNOLLE-MAinAR C., LÉNA J.-Y., CALVET A. (2014). « Concevoir le futur d'un territoire dans une perspective d'éducation au développement durable ». Vertigo - la revue électronique en sciences de l'environnement, vol. 14, $\mathrm{n}^{\circ} 1$. En ligne : http://journals.openedition.org/ vertigo/14690; DOI : 10.4000/vertigo.14690

LEFÈVRE L. (1947). « La construction d'un plan en relief en plâtre en $6^{\mathrm{e}}$ nouvelle ». L'Information géographique, vol. 11, n 5 p. 206-207. En ligne : https://www.persee.fr/doc/ ingeo_0020-0093_1947_num_11_5_5315

LEROYER L. (2005). "S'approprier le vocabulaire spatial et temporel par le "faire et le dire" ». Grand N, n 75, p. 31-43. En ligne : https://irem.univ-grenoble-alpes.fr/medias/fichier/75n4_1554803722209pdf

LÉVY J. (2019). « Capital spatial ». In LÉVY J. et LUSSAULT M. Dictionnaire de la géographie, Paris : Belin, p. $147-148$.

LUTZ W., MUTTARAK R., STRIESSNIG S. (2014). "Universal education is key to enhanced climate adaptation". Science, vol. 346, $\mathrm{n}^{\circ}$ 6213, p. 1061-1062.

MARTINAND J.-L. (1992). Enseignement et apprentissage de la modélisation en sciences. Paris : INRP.

MERLE H. (2000). « Comment aider à modéliser le ciel et la Terre ». Aster, nº 31, p. 37-70.

MUTTARAK R., LUTZ W. (2014). "Is education a key to reducing vulnerability to natural disasters and hence unavoidable climate change?". Ecology and Society, vol. 19, n 1, p. 1-8.

ORANGE C., FOURNEAU J.-C., BOURBIGOT J.-P. (2001). « Écrits de travail, débats scientifiques et problématisation à l'école élémentaire ». Aster, n 33, p. 111-133.

PARTOUNE C., ERICX M. (2005). « Les hyperpaysages. Comment percevoir, interpréter et exprimer la complexité des sociétés derrière les paysages ? ». Bulletin de la Société Géographique de Liège, vol. 45, p. 33-43.

PIERRARD M. A. (1988). « Modélisation et astronomie ». Aster, nº 7, p. 91-102.

ROMERO M., LILLE B., GIRARD M.-A., COHEN D., SPENCE Y. (2017). De Montréal à Antibes, apprentissages interdisciplinaires au secondaire par la construction de maquettes physico-numériques. Colloque de la CIRTA, Université du Québec à Montréal. En ligne : https://www.researchgate.net/profile/ Margarida_ROMERO/publication/

319211165_De_Montreal_a_Antibes_apprentissages_interdisciplinaires_au_secondaire_par_la_construction_de_maquettes_physiconumeriques/links/599c2a9945851574f4ac8dee/De-Montreal-a-Antibes-apprentissages-

interdisciplinaires-au-secondaire-par-la-construction-de-maquettes-physico-numeriques.pdf 
STRIESSNIG E., LUTZ W., PATT A. G. (2013). "Effects of educational attainment on climate risk vulnerability". Ecology and Society, vol. 18, $\mathrm{n}^{\circ}$ 1, p. 1-16.

TANNER T. (2010). "Shifting the narrative: Child-led responses to climate change and disasters in El Salvador and the Philippines". Children \& Society, vol. 24, n 4, p. 339-351.

VERGNOLLE MAINAR C., JULIEN M.-P., CHALMEAU R., CALVET A. et LÉNA J.-Y. (2016). « Recherches

collaboratives en éducation à l'environnement et au développement durable : l'enjeu de la modélisation de l'ingénierie éducative, pour une transférabilité d'un territoire à un autre ». Éducation Relative à l'Environnement, vol. 13, nº 1, p. 55-70. En ligne : https://ere.revues.org/333

WALLISER B. (1977). Systèmes et modèles. Paris : Le Seuil.

\section{NOTES}

1. http://www.memorisks.org

2. Il faut entendre réalité comme l'espace matériel, le topos (dimension physique d'un lieu, en opposition à la chôra soit la dimension sensible).

3. Ce travail a bénéficié d'une aide de l'État gérée par l'Agence Nationale de la Recherche au titre du Labex DRIIHM, programme «Investissements d'avenir » portant la référence ANR-11LABX-0010. Nous tenons également à remercier Régine Laurio et Jean-Daniel Cavaillé, professeurs des écoles des classes de $\mathrm{CP}-\mathrm{CE}$ et $\mathrm{CM}$, et leurs élèves.

4. Une enquête menée un an après auprès des élèves ayant participés à ce travail souligne une prise en compte plus aiguë des risques de crue qui étaient pourtant minorés au début de notre expérimentation.

\section{RÉSUMÉS}

La maquette est un outil efficient pour une éducation aux risques. En effet, elle peut favoriser la construction d'une culture du risque, la conscientisation de la vulnérabilité du territoire proche et vécu et la réflexion sur la résilience de la société. L'ingénierie éducative mise en place autour de l'élaboration de la maquette met en contact les élèves avec d'autres acteurs du territoire et elle développe les échanges et les débats. La maquette est donc, dans cette ingénierie, un outil heuristique, de prospective et de formation à la citoyenneté.

Scale models efficiently teach about risks. They can promote a culture of risk management, raise awareness of the vulnerability of nearby and lived-in territories, and encourage debate around societal resilience. The educational framework established around the building of a scale model brings together students and local stakeholders, increasing dialogue and debate. Thus, within this framework, scale models are a heuristic and prospective tool for citizenship education.

La maqueta es una herramienta eficaz para educar sobre la noción de riesgo. Desarrolla la cultura, valoriza la vulnerabilidad del territorio próximo y vivido, y ayuda a reflexionar sobre la capacidad de resiliencia de la sociedad ante el riesgo. Elaborando una maqueta se puede educar a los alumnos para conocer los actores que intervienen en el territorio $\mathrm{y}$, a la vez, es un instrumento heurístico, de prospectiva, de intercambio de ideas y de formación ciudadana. 
INDEX

Mots-clés : maquette, modèle, éducation aux risques, territoire, crue

Palabras claves : maqueta, modelo, educación sobre riegos, territorio, crecida

Keywords : scale model, model, risk education, territory, flood

\section{AUTEURS}

\section{DAVID BÉDOURET}

Laboratoire Géode, UMR CNRS 5602

RAPHAËL CHALMEAU

Laboratoire Géode, UMR CNRS 5602

CHRISTINE VERGNOLLE-MAINAR

Laboratoire Géode, UMR CNRS 5602

MARIE PIERRE JULIEN

Laboratoire Géode, UMR CNRS 5602

JEAN-YVES LÉNA

Laboratoire Géode, UMR CNRS 5602

ANNE CALVET

Laboratoire Géode, UMR CNRS 5602 